\title{
Spin pumping through a topological insulator probed by x-ray detected ferromagnetic resonance
}

\author{
A. I. Figueroa ${ }^{\mathrm{a}}$, A. A. Baker ${ }^{\mathrm{a}, \mathrm{b}}$, L. J. Collins-McIntyre ${ }^{\mathrm{b}}$, T. Hesjedal ${ }^{\mathrm{b}}$, G. van der Laan ${ }^{\mathrm{a}}$ \\ ${ }^{a}$ Magnetic Spectroscopy Group, Diamond Light Source, Didcot, OX11 ODE, United Kingdom \\ ${ }^{b}$ Department of Physics, Clarendon Laboratory, University of Oxford, Oxford, OX1 3PU, United Kingdom
}

\begin{abstract}
In the field of spintronics, the generation of a pure spin current (without macroscopic charge flow) through spin pumping of a ferromagnetic (FM) layer opens up the perspective for the new generation of dissipation-less devices. Microwave driven ferromagnetic resonance (FMR) can generate a pure spin current that enters adjacent layers, allowing for both magnetization reversal (through the spin transfer torque) and to probe the spin coherence properties of non-magnetic materials. However, standard FMR is unable to study multilayer dynamics directly, since the measurement averages over the contributions from the whole system. The synchrotron radiation-based technique of X-ray detected FMR (XFMR) offers an elegant solution to this drawback, giving access to element-, site-, and layer-specific dynamical measurements in heterostructures. In this work, we show how XFMR has provided unique information to understand spin pumping and spin transfer torque effects through a topological insulator (TI) layer in a pseudo-spin valve heterostructure. We demonstrate that TIs function as efficient spin sinks, while also allowing a limited dynamic coupling between ferromagnetic layers. These results shed new light on the spin dynamics of this novel class of materials, and suggest future directions for the development of room temperature TI-based spintronics.
\end{abstract}

Keywords: X-ray detected ferromagnetic resonance, spin pumping, spintronics, topological insulators

\section{Introduction}

Spin pumping has become a key effect in spintronic research in recent years [1,2] due to its promise of low-power data transmission in novel devices. Spin pumping refers to the phenomenon in which a precessing magnetic layer under ferromagnetic resonance (FMR) emits a spin current to an adjacent layer [3]. Such non-local spin dynamics manifest as an additional damping term in the Landau-Lifshitz-Gilbert (LLG) equation for magnetodynamics [4] broadening the measured resonance.

Conventional FMR measurements offer a natural way to study spin pumping effects in magnetic multilayers as it gives access to important parameters of the magnetization dynamics $[5,6,7,8,9]$. However, these methods are limited in that they can only detect the net response of the whole multilayer sample. Recently, x-ray detected FMR (XFMR) has emerged as a powerful synchrotron radiation based tool that can be used to study the element- (and thus layer-) specific magnetization dynamics [10, 11, 12, 13, 14, 15, 16, 17, 18, 19, 20, 21, 22, 23]. Magnetic and chemical contrast in XFMR is obtained by X-ray magnetic circular dichroism (XMCD) [24, 25]. The periodic oscillation of the magnetic moments under resonance is measured as a dynamic XMCD signal. The element specificity of XFMR offers clear advantages over FMR, in particular, it allows layer resolution of the magneto-dynamics in multilayer

Email address: aifigueg@gmail .com (A. I. Figueroa) samples. The application of this technique to study spin transfer effects, in particular of spin pumping through a nonmagnetic spacer, has demonstrated to be extremely useful as it provides layer-specific information of the phase and amplitude of precession of the individual ferromagnetic (FM) layers, thus allowing direct confirmation of the presence of a coherent pure spin current [26].

In this work, we use FMR and XFMR to study the dynamic spin transfer in a pseudo-spin valve heterostructure that incorporates a topological insulator (TI) as spacer layer. TIs have received widespread attention due to their large spin-orbit coupling and dissipationless, counterpropagating conduction channels in the surface state. In the prototypical three-dimensional $\mathrm{TI} \mathrm{Bi}_{2} \mathrm{Se}_{3}$ a large spin-orbit interaction leads to a band inversion in the bulk and the formation of a topologically protected surface state (TSS), with fully spin-polarised counter-propagating conduction channels that are robust against scattering from nonmagnetic impurities [27]. Figure 1(a) shows schematically the bandstructure of a TI. Spin-momentum locking allows the possibility of very long spin-flip scattering lifetimes and the ability to generate ultra-high spin-orbit torques [28, 29, 30]. It has been predicted that the TSS can exert a torque on spins in a neighbouring ferromagnet (FM) through exchange coupling [31]. The application of FMR to study the spin transfer dynamics through TIs is quite naturally suggested by the similarity between the spin-locked surface state of a TI and the separation of angular momentum and charge flow that takes place in a pure spin current. Recently, studies of such exciting effects have begun to emerge through electrical transport and inverse 
(a)

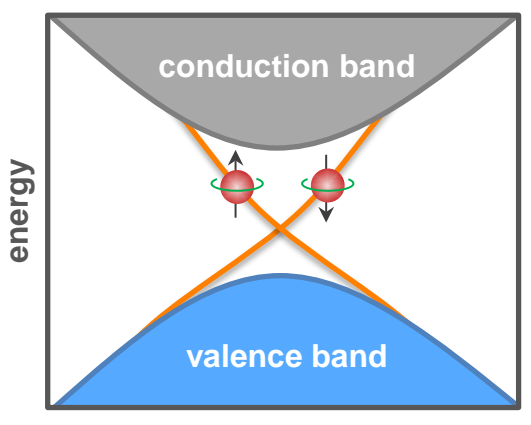

momentum (b)

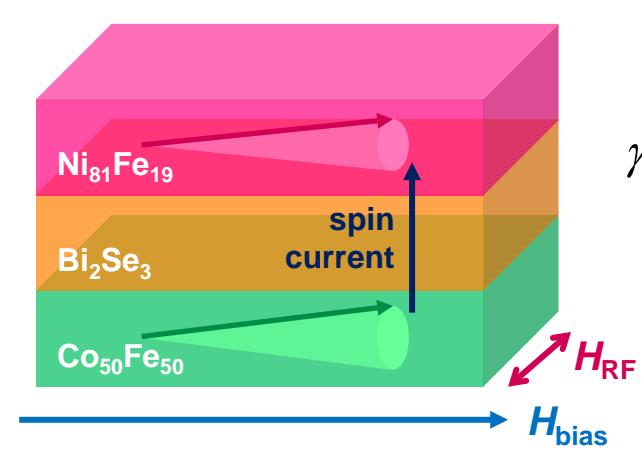

(c)

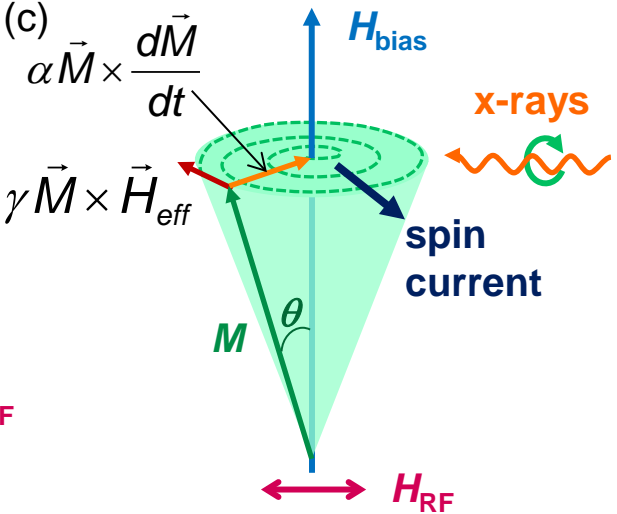

Figure 1: (a) Band structure of a bulk TI, depicting the valence (blue) and conduction (gray) bands, with the spin-locked surface state crossing the bulk bandgap. (b) Schematic of the FM/TI/FM heterostructure, showing the $\mathrm{TI}^{\mathrm{Bi}} \mathrm{Se}_{3}$ placed between two FM layers. The precession of magnetization excited around the static bias field drives a pure spin current from the $\mathrm{Co}_{50} \mathrm{Fe}_{50}$ through the $\mathrm{Bi}_{2} \mathrm{Se}_{3}$ into the $\mathrm{Ni}_{81} \mathrm{Fe}_{19}$, exerting a spin transfer torque. (c) Diagram of the forces acting on the magnetization of a material undergoing FMR, showing the excitation field $\left(H_{\mathrm{RF}}\right)$, static bias field $\left(H_{\text {bias }}\right)$ and precessing magnetization vector $(M)$. The direction of the incident $\mathrm{X}$-rays for XFMR in transverse geometry is also shown.

spin-hall effect measurements [32, 30, 33], demonstrating the great potential of TIs for incorporation into spintronic devices such as spin valves. With the present study, we demonstrate that TIs function as efficient spin sinks, while also allowing a limited dynamic coupling between ferromagnetic layers.

\section{Material and methods}

\subsection{Sample growth}

Samples were grown by molecular beam epitaxy, on $\mathrm{MgO}$ (001) substrates. Growth was monitored using in-situ reflection high energy electron diffraction (RHEED). A schematic of the heterostructure is shown in Fig. 1(b). First, $30 \mathrm{~nm}$ of $\mathrm{Co}_{50} \mathrm{Fe}_{50}$ is deposited and annealed to form an epitaxial ferromagnetic layer. Next, $\mathrm{Bi}_{2} \mathrm{Se}_{3}$ was deposited, with thickness ranging from $4 \mathrm{~nm}$ to $20 \mathrm{~nm}$. $30 \mathrm{~nm}$ of $\mathrm{Ni}_{81} \mathrm{Fe}_{19}$ is then deposited at room temperature $(300 \mathrm{~K})$ forming a polycrystalline layer. This is important in order to prevent damage to the TI due to Se outdiffusion or Fe intercalation. Further details about the growth of these heterostructures can be found in Ref. [34].

\subsection{VNA-FMR}

VNA-FMR measurements were performed using a Rhode and Schwartz ZVB20 vector network analyser in the frequency range 0.5-20 GHz. Samples were mounted face-down on a coplanar waveguide (CPW) of characteristic impedance $50 \Omega$ and placed in a vector electromagnet, capable of applying a field of up to $0.5 \mathrm{~T}$ in any direction. Real and imaginary components of the microwave transmission parameter, $S_{12}$, were measured as a function of field vector (strength and angle) and frequency. The resulting resonances were fitted using asymmetric Lorentzians to extract resonance frequency and linewidth. All measurements were performed at room temperature for varying bias field strength and angle with respect to the [100] axis of $\mathrm{Co}_{50} \mathrm{Fe}_{50}$, an in-plane hard axis.
Figure 1(c) shows the forces acting on the magnetization of a material undergoing FMR. An external bias field $H_{\text {bias }}$ is applied to align the magnetization $M$. A small transverse RF field $H_{\mathrm{RF}}$ drives the magnetization in precession about its axis. The precessional torque arises due to an effective internal field, $M \times H_{e f f}$, while $M \times(d M / d t)$ brakes the magnetization back towards the equilibrium condition according to the to the phenomenological Gilbert torque, $\alpha$. The Gilbert damping includes energy loss mechanisms such as spin-flip scattering and the excitation of phonons. In the classical limit the dynamics of magnetization can be described by the LLG equation of motion. In order to account for spin pumping mechanisms, this equation can be modified accordingly. For a trilayer structure with only one layer, $i$, on resonance, the motion of magnetization $\boldsymbol{m}_{i}$, is [2]:

$$
\frac{\partial \boldsymbol{m}_{i}}{\partial t}=-\gamma\left[\boldsymbol{m}_{i} \times \boldsymbol{H}_{\mathrm{eff}}^{i}\right]+\alpha_{i}^{0}\left[\boldsymbol{m}_{i} \times \frac{\partial \boldsymbol{m}_{i}}{\partial t}\right]+\alpha_{i}^{s p}\left[\boldsymbol{m}_{i} \times \frac{\partial \boldsymbol{m}_{i}}{\partial t}\right],
$$

where the subscript denotes the magnetic layer number, $\gamma$ is the gyromagnetic ratio, $\alpha^{0}$ the intrinsic Gilbert damping parameter and $\alpha^{\mathrm{sp}}$ additional damping due to spin pumping. The third term represents increased damping in layer $i$ due to spin pumping. If the second layer is allowed to precess an anti-damping (or accelerating) torque from the STT induced by momentum transfer from layer $j$ is also present, but this vanishes if the resonances are well separated in frequency-field space. This assumption is valid in all samples considered here, as confirmed by the XFMR measurements. The additional damping associated with spin pumping can be written as [26]:

$$
\alpha_{\mathrm{sp}}=\frac{g \mu_{\mathrm{B}}}{4 \pi M} \operatorname{Re}\left(\tilde{g}_{\uparrow \downarrow}\right) \frac{1}{d},
$$

where $g$ is the Landé $g$-factor, $\mu_{\mathrm{B}}$ the Bohr magneton, $M$ the magnetization of the magnetic layer, $d$ the layer thickness and 
$\tilde{g}_{\uparrow \downarrow}$ the spin mixing conductance, which controls spin-selective transport across the interface [1]. Spin pumping across a nonmagnetic barrier usually decays exponentially, as scattering of the pure spin current leads to a loss of angular momentum and backflow into the on-resonance layer. This is in effect a simultaneous damping and anti-damping of motion, for thinner barriers the second ferromagnet absorbs the spin current and minimizes backflow, while for thicker barriers spin pumping is reduced as more electrons are scattered.

\section{3. $X F M R$}

XFMR measurements were performed on beamline I10 at the Diamond Light Source, UK, and beamline 4.0.2 at the Advanced Light Source (ALS), US. XFMR offers an element (and thus layer-) specific time-resolved measurement of magnetization alignment, allowing mapping of the precessional dynamics of each FM layer on the picosecond timescale. The dynamical process of spin precession is detected using element-specific XMCD [35]. We recall that static XMCD is obtained as the difference between the two X-ray absorption spectra with the helicity vector of the circular polarization parallel and antiparallel respectively to an external magnetic field [25]. The XMCD signal is proportional to the projection of the helicity vector, which is along the beam direction $\hat{\mathbf{k}}$, onto the magnetization $\mathbf{M}$, hence $I_{\mathrm{XMCD}} \propto \hat{\mathbf{k}} \cdot \mathbf{M}$. The same XMCD spectrum can be obtained by reversing either the magnetic field or the circular polarization direction in the experimental geometry. In the XFMR technique, we use the XMCD effect to measure with fixed circular polarization the small periodic oscillations in the magnetization direction.

The sample is mounted on a CPW and driven by an applied RF field while under a DC bias field. A schematic of the experimental setup for XFMR can be seen in Fig. 2 (see also references [17, 19, 20, 36] for alternative approaches). Both at Diamond and the ALS, the bias field was supplied using a vector magnet, in which the field can be applied in any direction up to $0.5 \mathrm{~T}$ [37]. The vector magnet is particularly suitable for XFMR as it allows a simple change of $H_{\text {bias }}$ from $(i)$ parallel to the photon direction, which is needed for static XMCD scans to (ii) orthogonal to both the photon direction and the RF excitation, required for phase-resolved XFMR. The vector electromagnet design consists of eight current coils, positioned externally along the three-fold axes of the six-way-cross vacuum chamber, which allows free access for the $\mathrm{x}$-ray beam and the sample manipulator.

Two main different methods can be used to measure XFMR, namely time-averaged using a longitudinal geometry [11, 15] and time-resolved using a transverse geometry [19]. In timeaveraged XFMR, the shortening of the magnetization vector along the $z$-axis (x-rays direction) leads to a difference $\Delta M_{z}=$ $M_{0}(1-\cos \theta) \approx \frac{1}{2} M_{0} \theta^{2}$, where $\theta$ is the cone angle of the Larmor precession. The time-averaged XFMR requires no synchronization with the synchrotron, which means it can be measured at any frequency, provided the RF power is sufficiently strong.

In this study, we use the time-resolved mode for XFMR, which gives access to the precessional phase. With the bias field perpendicular to the incident $\mathrm{x}$-ray beam, the oscillating component of the Larmor precession is observed with a magnitude $\left|M_{y}\right|=M_{0} \sin \theta \approx M_{0} \theta$. Thus the XFMR signal in transverse geometry has the advantage that it is larger than in longitudinal geometry by a factor $2 \theta^{-1}$. At the resonance frequency the cone angle $\theta$ is typically in the order of $10 \mathrm{mrad}\left(\approx 0.57^{\circ}\right)$, $[14,38]$, resulting in a dynamic XMCD in transverse and longitudinal geometry of $1 \%$ and $0.005 \%$, respectively, of the static $\mathrm{XMCD}$. The precession angle is strongly elliptical due to the shape anisotropy of the film. We can expect a larger amplitude in the plane $\left(M_{y}\right)$ than normal to the plane $\left(M_{x}\right)$, hence this favours a measurement geometry where the X-rays are incident at grazing angle. The time-resolved XFMR is detected stroboscopically by using the periodic $\mathrm{x}$-ray pulses from the synchrotron. The RF is fixed to a multiple of the $\mathrm{x}$-ray pulse frequency (499.65 MHz) and the applied field can be used to tune the resonance.

For the stroboscopic measurements, the RF has to be a harmonic of X-ray pulse frequency, hence the resonance is driven at multiples of the master oscillator clock of the storage ring. These higher harmonics are generated using an RF comb generator (Atlantic Microwave) driven by the master oscillator, which has a frequency of $499.65 \mathrm{MHz}$, both at Diamond and the ALS, corresponding to $\sim 2 \mathrm{~ns}$ intervals between consecutive $\mathrm{X}$-ray pulses. The desired frequency is selected using filters and amplifiers to drive a narrow band, high power (25-30 dBm) RF field to the CPW. A programmable delay line (Colby Instruments) enables phase shifting of the RF oscillation with respect to the $\mathrm{x}$-ray pulses with a step resolution of $\sim 0.5 \mathrm{ps}$.

Incident $\mathrm{X}$-rays impinge the sample through the hole on the signal line of the CPW. The $\mathrm{x}$-ray beam has an incidence angle of $35^{\circ}$ with respect to the plane of the sample, to ensure that the XMCD is sensitive to the larger in-plane component of the magnetization precession. The X-ray absorption was measured in transmission geometry by detecting the $\mathrm{x}$-ray excited optical luminescence emerging from the $\mathrm{MgO}$ substrate [39], using a photodiode mounted behind the sample. Lock-in detection was employed to measure the small amplitude precession using a lock-in amplifier (LIA) while modulating the phase of the driving RF field through $180^{\circ}$. All XFMR measurements were performed at the $\mathrm{Ni}$ and $\mathrm{Co} L_{3}$ edges.

\section{Results and discussion}

A field-frequency map obtained from the VNA-FMR measurements on a representative sample $\left(t_{\mathrm{TI}}=4 \mathrm{~nm}\right)$ is shown in Fig. 3. The resonance angular frequency, $\omega=2 \pi f$, for in-plane magnetization is determined by the Kittel equation [40], as 


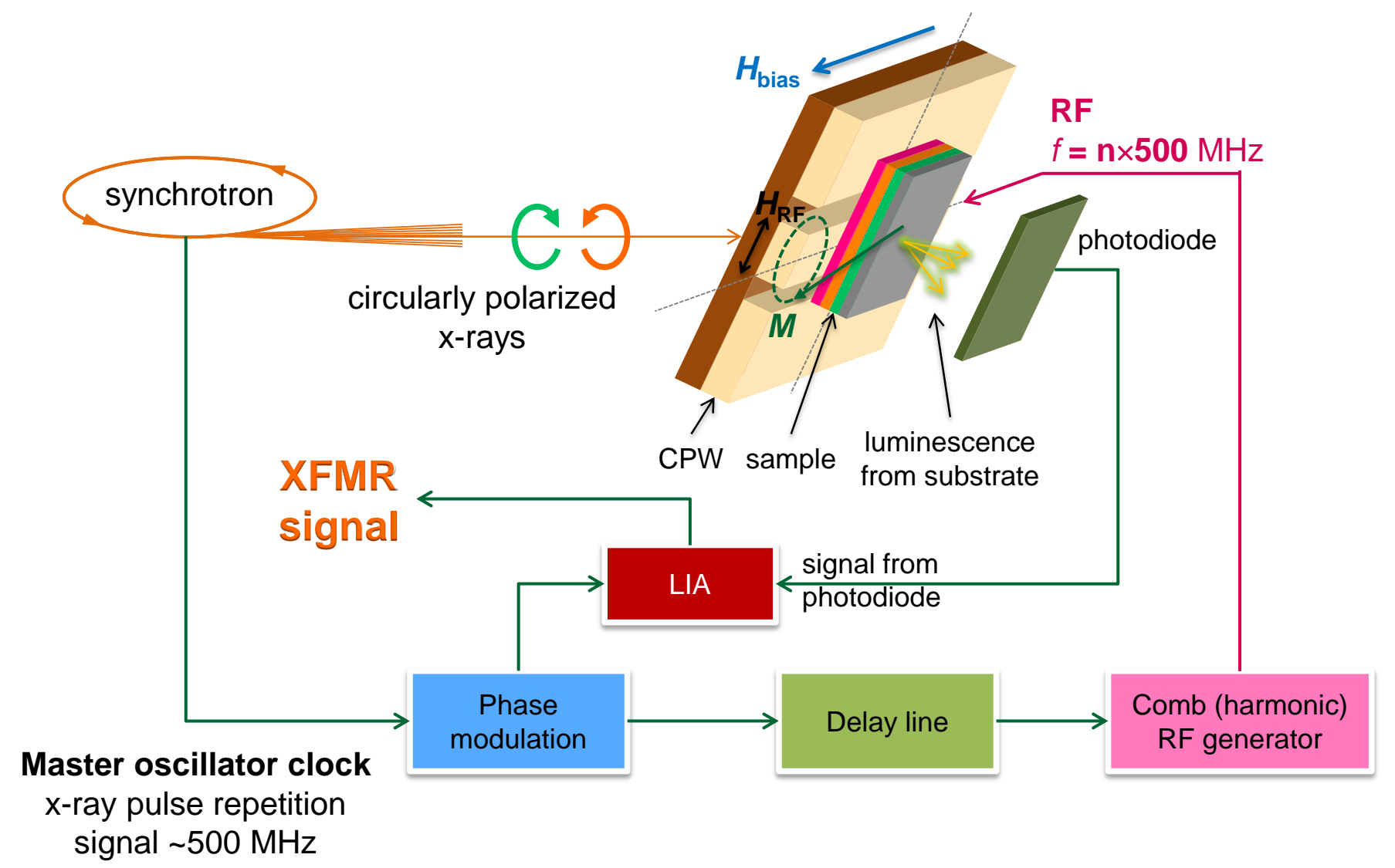

Figure 2: Schematics of the XFMR setup in transverse geometry used for the layer specific characterization of the magnetization dynamics of the FM/TI/FM heterostructure. The sample is placed face-down on a CPW (within the vector magnet that provides the magnetic field) and incident circularly polarized x-rays pass through a hole in the signal line. The magnetization of the stack about $H_{\text {bias }}$ is driven by the RF field, $H_{\mathrm{RF}}$. The cone angle of precession is exaggerated for clarity; the typical magnitude of precession is $\sim 1^{\circ}$. The applied field $H_{\text {bias }}$ is perpendicular to the x-ray beam direction and along the $\mathrm{CPW}$ transmission line. The different components of the XFMR electronic signal circuitry, RF excitation circuit, and x-ray detection system are also shown. The delay line enables phase shifting of the $\mathrm{RF}$ oscillation with respect to the $\mathrm{x}$-ray pulses with $0.5 \mathrm{ps}$ step resolution.

$$
\begin{aligned}
\left(\frac{\omega}{\gamma}\right)^{2}= & {\left[\mu_{0} M+\mu_{0} H \cos \Delta \phi+\frac{K_{\mathrm{c} \|}}{2 M}\left\{3+\cos 4\left(\Delta \phi+\phi_{\mathrm{H}}\right)\right\}\right.} \\
& \left.+\frac{K_{\mathrm{u} \|}}{M}\left\{1-\cos 2\left(\Delta \phi+\phi_{\mathrm{H}}-\phi_{\mathrm{u}}\right)\right\}\right] \\
& \times\left[\mu_{0} H \cos \Delta \phi+\frac{2 K_{\mathrm{c} \|}}{M}\left\{\cos 4\left(\Delta \phi+\phi_{\mathrm{H}}\right)\right\}\right. \\
& \left.-\frac{2 K_{\mathrm{u} \|}}{M} \cos 2\left(\Delta \phi+\phi_{\mathrm{H}}\right)\right]
\end{aligned}
$$

with $\gamma$ the gyromagnetic ratio, $\mu_{0} H$ the Zeeman field, $\Delta \phi$ the canting of magnetization away from the bias field, $K_{\mathrm{c} \|}$ and $K_{\mathrm{u} \|}$ the cubic and uniaxial anisotropy parameter, respectively, and $\phi_{\mathrm{H}}$ and $\phi_{\mathrm{u}}$ the angle of the bias field and uniaxial anisotropy, with respect to the [100] axis of $\mathrm{Co}_{50} \mathrm{Fe}_{50}$, respectively. Simultaneous fitting of the field and angle dependence of the resonance yielded the parameters listed in Table 1.

The linewidth of the resonance is frequency $(f)$ dependent and relates to the damping as [41]

\begin{tabular}{cccccc}
\hline \hline & $\begin{array}{c}t_{\mathrm{TI}} \\
{[\mathrm{nm}]}\end{array}$ & $\begin{array}{c}K c_{\|} \\
{\left[\mathrm{kJ} \mathrm{m}^{-3}\right]}\end{array}$ & $\begin{array}{c}K u_{\|} \\
{\left[\mathrm{kJ} \mathrm{m}^{-3}\right]}\end{array}$ & $g$ & $\begin{array}{c}M_{\mathrm{s}} \\
{\left[\mathrm{MA} \mathrm{m}^{-1}\right]}\end{array}$ \\
\hline \multirow{6}{*}{$\mathrm{Co}_{50} \mathrm{Fe}_{50}$} & 4 & $41.9 \pm 0.2$ & $3.5 \pm 0.1$ & 2.10 & 1.76 \\
& 8 & $43.0 \pm 0.1$ & $0.94 \pm 0.08$ & 2.09 & 1.76 \\
& 20 & $27.5 \pm 0.7$ & $2.78 \pm 0.06$ & 2.11 & 1.76 \\
\hline $\mathrm{Ni}_{81} \mathrm{Fe}_{19}$ & 6 & $2.19 \pm 0.04$ & $2.19 \pm 0.04$ & 1.91 & 0.96 \\
& 8 & $0.16 \pm 0.06$ & $0.87 \pm 0.05$ & 1.90 & 0.96 \\
& 20 & $0.82 \pm 0.04$ & $1.21 \pm 0.05$ & 1.92 & 0.96 \\
\hline \hline
\end{tabular}

Table 1: Magnetocrystalline anisotropy parameters and $g$-factor for the $\mathrm{Co}_{50} \mathrm{Fe}_{50}$ and $\mathrm{Ni}_{81} \mathrm{Fe}_{19}$ modes of the heterostructures, determined by fitting of angle- and field-dependent FMR frequency for multilayered samples with indicated TI thickness, $t_{\mathrm{TI}}$.

$$
\Delta H=\Delta H_{0}+\frac{4 \pi \alpha f}{|\gamma|},
$$

where $\Delta H$ is the full width at half maximum of the resonance, 
$\Delta H_{0}$ the inhomogeneous broadening arising from non-Gilbert damping mechanisms such as lattice defects and $\alpha=\alpha^{0}+\alpha^{\mathrm{sp}}$ the Gilbert damping, including contributions related to coupling to the lattice (such as spin flip scattering, phonon drag, or spin pumping).

Damping in trilayers normally drops exponentially with spacer thickness [42], as scattering within the non-magnetic (NM) layer is a much less efficient sink for angular momentum than absorption by a second ferromagnet. In our heterostructures, however, damping increases with TI thickness, as depicted in Fig. 3(b), where the dependence of the measured damping parameter, $\alpha$, for the $\mathrm{Ni}_{81} \mathrm{Fe}_{19}$ and $\mathrm{Co}_{50} \mathrm{Fe}_{50}$ layers as a function of the thickness of the TI interlayer is shown. A linear increase is observed up to $20 \mathrm{~nm}$, wherein the spin pumping component represents a significant fraction of the total damping, indicating a large transfer of angular momentum to the TI. These results suggest not only that the TI is a very efficient spin sink, but that the pure spin current can penetrate some distance into the TI, at least $8 \mathrm{~nm}$ (compared to $\sim 3 \mathrm{~nm}$ in Si or $<1 \mathrm{~nm}$ in Ta $[43,26]$ ).

As a spin current is driven into the TSS a spin imbalance develops, which is converted to a charge current [30]. The scattering of such conduction electrons within the bulk of the TI could then provide a mechanism for efficient absorption of pumped angular momentum.

It is instructive to analyse the spin pumping results in the analytical framework of the STT in normal metals. Equation 2 can be rearranged to yield the spin mixing conductance, $\tilde{g}_{\uparrow \downarrow}$. Considering, for example, the case of the $\mathrm{Ni}_{81} \mathrm{Fe}_{19}\left(\mathrm{M}_{s}=0.906\right.$ $\times 10^{6} \mathrm{~A} \mathrm{~m}^{-1}$ ) layer in the $t_{\mathrm{TI}}=20 \mathrm{~nm}$ sample, the spin pumping damping can be extracted by comparison with a bare $\mathrm{Ni}_{81} \mathrm{Fe}_{19}$ layer, yielding $\alpha_{\mathrm{sp}}=(2.6 \pm 0.3) \times 10^{-3}$. The spin mixing conductance is then $\tilde{g}_{\uparrow \downarrow}=(4.2 \pm 0.5) \times 10^{15} \mathrm{~cm}^{-2}$. Performing the same calculation for the $\mathrm{Co}_{50} \mathrm{Fe}_{50}$ layer in this sample $\left(M_{s}\right.$ $\left.=1.76 \times 10^{6} \mathrm{~A} \mathrm{~m}^{-1}\right)$ gives $\tilde{g}_{\uparrow \downarrow}=(2.49 \pm 0.1) \times 10^{15} \mathrm{~cm}^{-2}$. The discrepancy between the results for the two layers points towards dissimilar interfaces. This most likely arises due to the requirement for low temperature deposition of the $\mathrm{Ni}_{81} \mathrm{Fe}_{19}$ layer, in order to preserve crystal quality within the $\mathrm{Bi}_{2} \mathrm{Se}_{3}$ layer.

Since the damping does not completely saturate these value should be regarded only as a lower limit on the effective spin mixing conductance available in FM/TI heterostructures. These values are comparable to the spin mixing conductances calculated by Jamali et al. [44] from their inverse spin Hall effect measurements. They are greater than previous reports for even a good spin conductor such as Ag, where $\tilde{g}_{\uparrow \downarrow} \approx 2 \times 10^{15} \mathrm{~cm}^{-2}$ [45]. Note that the calculation does not separate pumping into the bulk and surface state, which display very different spin dynamics. The value should therefore be considered only as a rough estimate of the effective spin mixing conductance that is available in TI heterostructures.

XFMR measurements allow for layer-specific determination of the phase and amplitude of precession, making it a powerful tool to study the subtle effects of coupling through the TI. Results obtained for the precession of Ni magnetization at $4 \mathrm{GHz}$ are shown in Fig. 4(a) for the $\mathrm{Co}_{50} \mathrm{Fe}_{50}(30) / \mathrm{Bi}_{2} \mathrm{Se}_{3}(8) /$ $\mathrm{Ni}_{81} \mathrm{Fe}_{19}(30)$ sample (thicknesses in $\mathrm{nm}$ ). The increase in am-
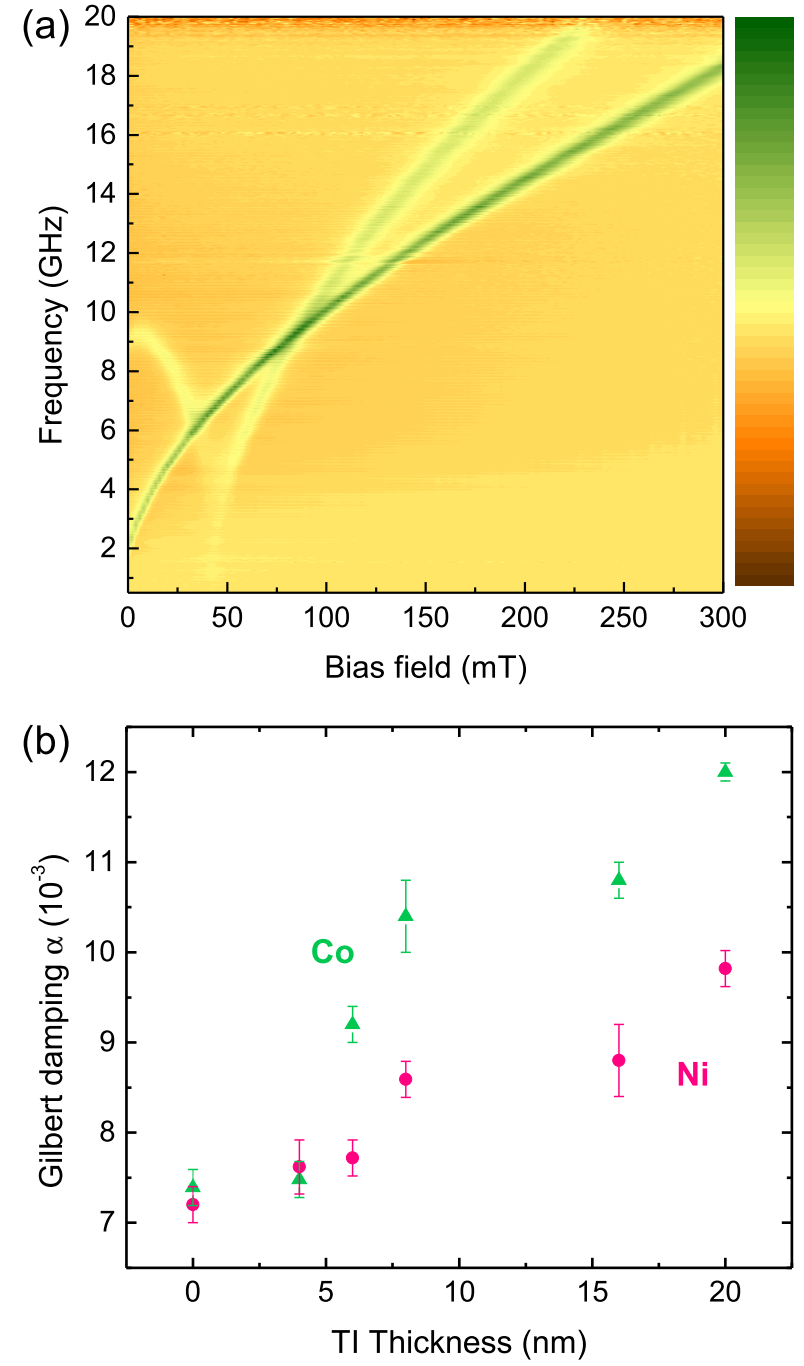

Figure 3: (a) Field versus frequency transmission map showing on-resonance absorption of the heterostructure. Green indicates high absorption, when the resonance condition is met. The bias field is applied parallel to the [100] axis of $\mathrm{Co}_{50} \mathrm{Fe}_{50}$, an in-plane hard axis, so the lower branch of the Kittel curve corresponds to magnetization canted along the in-plane easy axis, the upper branch to the magnetization collinear with the bias field. (b) Calculated damping factor as a function of thickness of the TI interlayer for $\mathrm{Co}_{50} \mathrm{Fe}_{50}$ (green) and $\mathrm{Ni}_{81} \mathrm{Fe}_{19}$ (pink) layers. Error bars represent the uncertainty on linear fits to linewidth as a function of frequency. 
plitude of precession and phase shift is clearly visible across the resonant field of $14 \mathrm{mT}$. Fitting sine curves to the precession yields amplitude and phase, which allows study of the coupling of the two layers.

The amplitude and phase of precession for Co and Ni determined in this way for two different thicknesses of TI interlayer are shown in Fig. 4(b-e). In a traditional spin valve, coupling of the two magnetic layers is mediated by the passage of a pure spin current through the spacer, leading to off resonance precession and a phase shift. Such features can be seen in the $\mathrm{Ni}_{81} \mathrm{Fe}_{19}$ layer for $t_{\mathrm{TI}}=8 \mathrm{~nm}$ (Fig. 4(c)) and the $\mathrm{Co}_{50} \mathrm{Fe}_{50}$ layer for $t_{\mathrm{TI}}=4 \mathrm{~nm}$ (Fig. 4(b)). However, this effect is rather weak, suggesting a suppression of spin pumping due to spinflip scattering. Measurements on a sample with $20 \mathrm{~nm} \mathrm{Bi}{ }_{2} \mathrm{Se}_{3}$ (not shown) showed no evidence of coupling, suggesting complete absorption of the pumped spin angular momentum. This confirms that the weak dynamic coupling of the two layers is suppressed at this thickness, suggesting that the pumped spin current is scattered by the maximum thickness measured. We note that good conductors, such as $\mathrm{Cu}, \mathrm{Ag}$ or $\mathrm{Au}$, can have a spin coherence length of tens of nanometres [42], while trivial insulators such as $\mathrm{MgO}$ or heavy metals such as Ta suppress spin pumping after just a few nm $[46,26]$. Suppression of spin pumping in insulators was recently studied by Du et al. [43], who showed that there was a characteristic decay length of under $1 \mathrm{~nm}$ for $\mathrm{SrTiO}_{3}, \mathrm{Sr}_{2} \mathrm{GaTaO}_{6}$, and $\mathrm{Sr}_{2} \mathrm{CrNbO}_{6}$. Amorphous silicon also suppressed spin pumping for films $3 \mathrm{~nm}$ thick. As a heavy element bulk bandgap insulator $\mathrm{Bi}_{2} \mathrm{Se}_{3}$ might therefore be expected to suppress spin pumping, absorbing angular momentum.

The XFMR results must therefore be considered alongside the VNA-FMR measurements. First, it is important to consider the distinction between the two techniques: VNA-FMR measures increased damping due to spin pumping out of a FM, while XFMR allows detection of modified precessional dynamics induced by spin pumping into a FM. The increase of damping parameter with TI thickness suggests that the TI functions as an excellent spin sink, but transfer of the spin current is less efficient. The weak coupling observed in the XFMR could be attributed to transmission of a pure spin current, with a decay length on the order of $8 \mathrm{~nm}$. While the current can persist within the TI (as demonstrated by continued increase of Gilbert damping with TI thickness), its passage across the FM/TI interface and the topological surface state is suppressed. The dissimilar interfaces indicated by the calculated values of $\tilde{g}_{\uparrow \downarrow}$ suggest that this transmission could be improved (and even higher values of $\tilde{g}_{\uparrow \downarrow}$ obtained) if the quality of the interface in future devices can be improved.

An alternative explanation could arise from the proximity coupling of 'top' and 'bottom' surface states that takes place in ultrathin TIs. As shown by Zhang et al. [47], for $\mathrm{Bi}_{2} \mathrm{Se}_{3}$ thicknesses below $6 \mathrm{~nm}$ the surface state at the two interfaces can interfere, disrupting the bandstructure. In this instance, direct communication between top and bottom surface should be possible, without passing through the bulk. If the TSS at each surface in these samples have retreated by $\sim 1 \mathrm{~nm}$ due to FM proximity or surface roughness [48], then this could explain the coupling observed for $4 \mathrm{~nm}$ and $8 \mathrm{~nm}$, while the distinct states at $20 \mathrm{~nm}$ do not allow this. The VNA-FMR experiments then probe the spin sink properties of the bulk, which has great capacity to absorb angular momentum, arising from its large spin-orbit coupling.

\section{Conclusion}

XFMR constitutes a powerful experimental technique to study magnetization dynamics, in particular the spin pumping phenomenon. It provides unique information about the spin dynamics of complex layer stacks and the spin transfer between magnetic layers through interfaces. By combining VNA-FMR and XFMR we have demonstrated that TIs hold great promise for the field of spintronics. We have shown that TIs posses a high capacity to absorb angular momentum, and-if the process is reversed-to generate a significant spin transfer torque. Our results reveal that transfer of angular momentum between ferromagnetic layers in a spin valve structure is possible, either through conventional spin pumping or possibly through a direct coupling of the topological surface state. It is important to highlight that all this findings have been observed at room temperature, which is promising for the incorporation of TIs in electronic and spintronic devices.

\section{Acknowledgements}

We thank Diamond Light Source for beamtime on I10 and the Advanced Light Source for beamtime on 4.0.2 (Magnetic Spectroscopy), as well as the Research Complex at Harwell for their hospitality. A.A.B. acknowledges funding from Diamond Light Source through a joint studentship and Wadham College through a senior scholarship. A.A.B. and L.C.M. acknowledge support from the EPSRC through doctoral training awards. Funding from the John Fell Oxford University Press Research Fund is acknowledged.

\section{References}

[1] Y. Tserkovnyak, A. Brataas, G. E. Bauer, B. I. Halperin, Rev. Mod. Phys. 77 (2005) 1375.

[2] B. Heinrich, Y. Tserkovnyak, G. Woltersdorf, A. Brataas, R. Urban, G. E. Bauer, Phys. Rev. Lett. 90 (2003) 187601.

[3] A. Brataas, Y. Tserkovnyak, G. E. W. Bauer, B. I. Halperin, Phys. Rev. B 66 (2002) 060404

[4] Y. Tserkovnyak, A. Brataas, G. E. Bauer, Phys. Rev. B 66 (2002) 224403.

[5] J. F. Cochran, B. Heinrich, A. S. Arrott., Phys. Rev. B 34 (1986) 77887801.

[6] P. J. Metaxas, R. L. Stamps, J. P. Jamet, J. Ferre, B. R. V. Baltz, P. Politi., Phys. Rev. Lett. 104 (2010) 237206.

[7] R. Magaraggia, K. Kennewell, M. Kostylev, R. L. Stamps, M. Ali, D. Greig, B. J. Hickey, C. H. Marrows., Phys. Rev. B 83 (2011) 054405.

[8] A. M. Kaiser, C. Schoeppner, F. M. Roemer, C. Hassel, C. Wiemann, S. Cramm, F. Nickel, P. Grychtol, C. Tieg, J. Lindner, C. M. Schneider., Phys. Rev. B 84 (2011) 134406.

[9] A. A. Timopheev, Y. G. Pogorelov, S. Cardoso, P. P. Freitas, G. N. Kakazei, N. A. Sobelev., Phys. Rev. B 89 (2014) 144410.

[10] T. Martin, G. Woltersdorf, C. Stamm, H. A. Dürr, R. Mattheis, C. H. Back, G. Bayreuther., J. Appl. Phys. 103 (2008) 07B112.

[11] J. Goulon, A. Rogalev, F. Wilhelm, N. Jaouen, C. Goulon-Ginet, G. Goujon, J. Ben Youssef, M. V. Indendom., JETP Lett. 82 (2005) 696-701. 
(a)

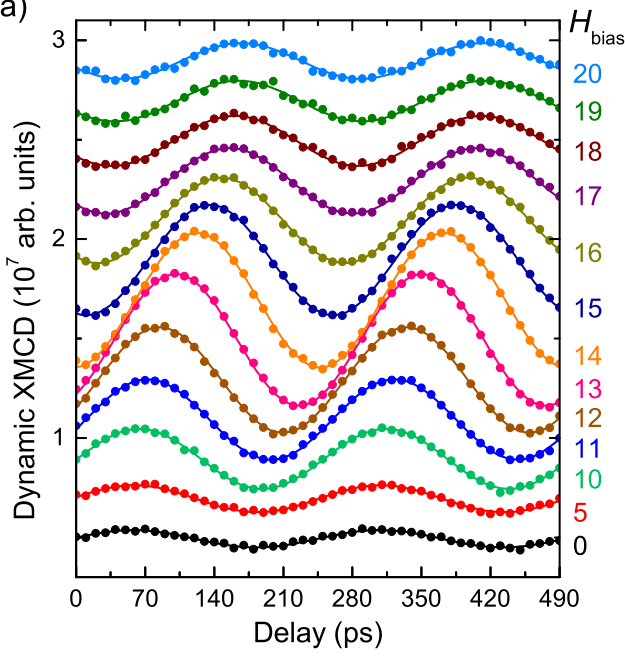

$(\mathrm{mT})$

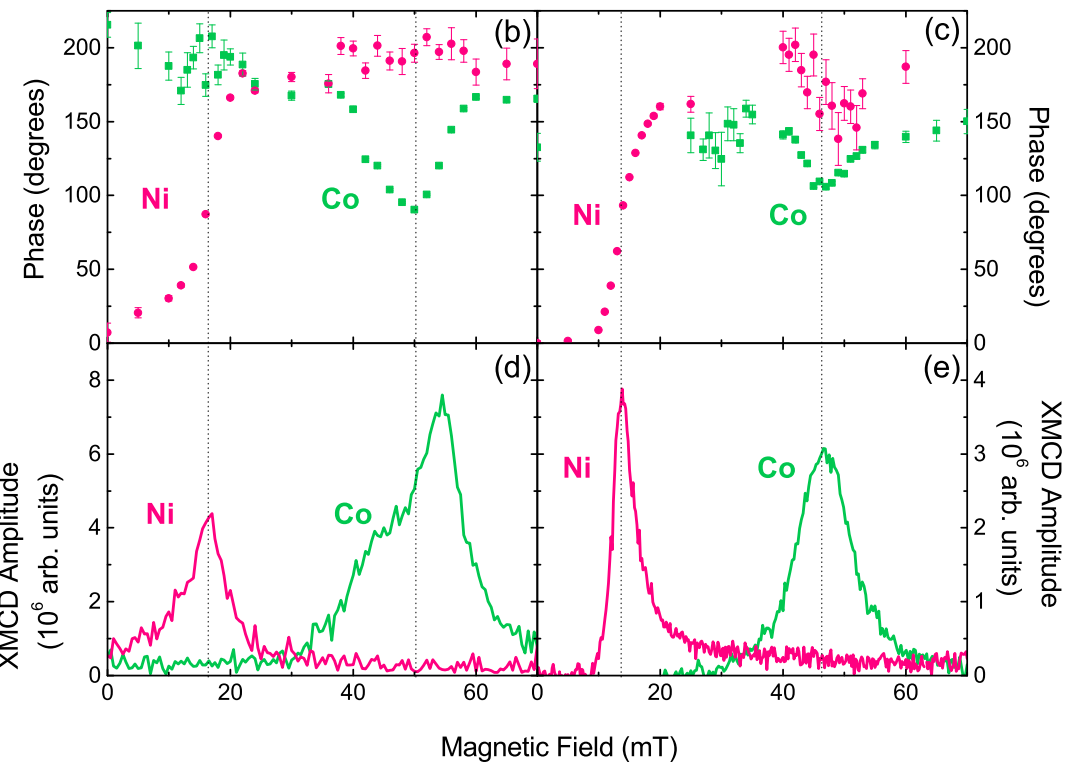

Figure 4: (a) XFMR data for $\mathrm{Ni}_{81} \mathrm{Fe}_{19}$ continuously driven at $4 \mathrm{GHz}$ for the $\mathrm{Co}_{50} \mathrm{Fe}_{50}(30) / \mathrm{Bi}_{2} \mathrm{Se}_{3}(8) / \mathrm{Ni}_{81} \mathrm{Fe}_{19}(30)$ sample (thicknesses in nm). Varying static bias field indicated by color of the lines, showing an increase in amplitude and phase shift across resonance at 14 mT. Solid lines are sine curve fits to the data. Curves have been vertically shifted for clarity. (b,c) Phase and (d,e) amplitude of precession of magnetization for $\mathrm{Ni}_{81} \mathrm{Fe}_{19}$ (pink circles) and Co ${ }_{50} \mathrm{Fe}_{50}\left(\mathrm{green}_{2}\right.$ triangles) layers at $4 \mathrm{GHz}$ driving frequency for $t_{\mathrm{TI}}=4 \mathrm{~nm}$ and $8 \mathrm{~nm}$, respectively. Dashed lines show the positions of the resonance amplitude peak. The drop and recovery in phase across the Co mode is caused by the superposition of the two modes, corresponding to canted and collinear magnetization, as can be seen in Fig. 3(a). Error bars in (b) and (c) arise from uncertainty on fits to the time-resolved precession in (a).

[12] J. Goulon, A. Rogalev, F. Wilhelm, N. Jaouen, C. Goulon-Ginet, C. Brouder., Eur. Phys. J. B 53 (2006) 169-184.

[13] Y. Guan, W. E. Bailey, C.-C. Kao, E. Vescovo, D. A. Arena., J. Appl. Phys. 99 (2006) 08J305.

[14] Y. Guan, W. E. Bailey, E. Vescovo, C.-C. Kao, D. A. Arena., J. Magn. Magn. Mater. 312 (2007) 437-378.

[15] G. Boero, S. Mouaziz, S. Rusponi, P. Bencok, F. Nolting, S. Stepanow, , P. Gambardella., New J. Phys. 10 (2008) 013011.

[16] G. Boero, S. Rusponi, P. Bencok, R. Meckenstock, J.-M. Thiele, F. Nolting, P. Gambardella., Phys. Rev. B 79 (2009) 224425.

[17] G. Boero, S. Rusponi, J. Kavich, A. Lodi Rizzini, C. Piamonteze, F. Nolting, C. Tieg, J.-U. Thiele, P. Gambardella., Rev. Sci. Instrum. 80 (2009) 123902.

[18] J. Goulon, A. Rogalev, F. Wilhelm, G. Goujon, C. Brouder, A. Yaresko, J. Ben Youssef, M. V. Indenbom., J. Magn. Magn. Mater. 322 (2010) 2308-2329.

[19] D. A. Arena, Y. Ding, E. Vescovo, S. Zohar, Y. Guan, W. E. Bailey., Rev. Sci. Instrum. 80 (2009) 083903.

[20] M. K. Marcham, P. S. Keatley, A. Neudert, R. J. Hicken, S. A. Cavill, L. R. Shelford, G. van der Laan, N. D. Telling, J. R. Childress, J. A. Katine, P. Shafer, E. Arenholz, J. Appl. Phys. 109 (2011) 07D353.

[21] M. K. Marcham, L. R. Shelford, S. A. Cavill, P. S. Keatley, W. Yu, P. Shafer, A. Neudert, J. R. Childress, J. A. Katine, E. Arenholz, N. D. Telling, G. van der Laan, R. J. Hicken, Phys. Rev. B 87 (2013) 180403(R).

[22] W. E. Bailey, C. Cheng, R. Knut, O. Karis, S. Auffret, S. Zohar, D. Keavney, P. Warnicke, J.-S. Lee, D. A. Arena., Nat. Commun. 4 (2013) 2025.

[23] M. K. Marcham, W. Yu, P. S. Keatley, L. R. Shelford, P. Shafer, S. A. Cavill, H. Qing, A. Neudert, J. R. Childress, J. A. Katine, E. Arenholz, N. D. Telling, G. van der Laan, , R. J. Hicken, Appl. Phys. Lett. 102 (2013) 062418

[24] G. van der Laan, B. T. Thole., Phys. Rev. B 43 (1991) 13401-13411.

[25] G. van der Laan., J. Phys.: Conf. Ser. 430 (2013) 012127.

[26] M. Marcham, L. Shelford, S. Cavill, P. Keatley, W. Yu, P. Shafer, A. Neudert, J. Childress, J. Katine, E. Arenholz, et al., Phys. Rev. B 87 (2013) 180403.

[27] J. E. Moore, Nature 464 (2010) 194-198.

[28] M. H. Fischer, A. Vaezi, A. Manchon, E.-A. Kim, arXiv preprint
arXiv:1305.1328 (2013).

[29] Y. Fan, P. Upadhyaya, X. Kou, M. Lang, S. Takei, Z. Wang, J. Tang, L. He, L.-T. Chang, M. Montazeri, et al., Nature Mater. 13 (2014) 699-704.

[30] Y. Shiomi, K. Nomura, Y. Kajiwara, K. Eto, M. Novak, K. Segawa, Y. Ando, E. Saitoh, Phys. Rev. Lett. 113 (2014) 196601.

[31] T. Yokoyama, J. Zang, N. Nagaosa, Phys. Rev. B 81 (2010) 241410.

[32] J. Tian, I. Childres, H. Cao, T. Shen, I. Miotkowski, Y. P. Chen, Solid State Commun. 191 (2014) 1-5.

[33] A. Mellnik, J. Lee, A. Richardella, J. Grab, P. Mintun, M. Fischer, A. Vaezi, A. Manchon, E.-A. Kim, N. Samarth, et al., Nature 511 (2014) 449.

[34] A. A. Baker, A. I. Figueroa, L. J. Collins-McIntyre, G. van der Laan, T. Hesjedal, Sci. Rep 5 (2015) 7907.

[35] G. van der Laan, A. I. Figueroa., Coord. Chem. Rev. $277-278$ (2014) 95129.

[36] D. A. Arena, E. Vescovo, C. C. Kao, Y. Guan, W. E. Bailey., Phys. Rev. B 74 (2006) 064409.

[37] E. Arenholz, S. O. Prestemon., Rev. Sci. Instrum. 76 (2005) 083908.

[38] O. Mosendz, J. E. Pearson, F. Y. Fradin, G. E. W. Bauer, S. D. bader, A. Hoffmann., Phys. Rev. Lett. 104 (2010) 046601

[39] C. A. F. Vaz, C. Moutafis, M. Buzzi, J. Raabe., J. Electron Spectrosc. Relat. Phenom. 189 (2013) 1-4.

[40] M. Farle, Rep. Prog. Phys. 61 (1998) 755.

[41] S. S. Kalarickal, P. Krivosik, M. Wu, C. E. Patton, M. L. Schneider, P. Kabos, T. Silva, J. P. Nibarger, J. Appl. Phys. 99 (2006) 093909.

[42] B. Kardasz, B. Heinrich, Phys. Rev. B 81 (2010) 094409.

[43] C. Du, H. Wang, Y. Pu, T. Meyer, P. Woodward, F. Yang, P. Hammel, Phys. Rev. Lett. 111 (2013) 247202.

[44] M. Jamali, J. S. Lee, Y. Lv, Z. Zhao, N. Samarth, J.-P. Wang, arXiv preprint arXiv:1407.7940 (2014).

[45] J. R. Sánchez, L. Vila, G. Desfonds, S. Gambarelli, J. Attané, J. De Teresa, C. Magén, A. Fert, Nat. Commun. 4 (2013).

[46] O. Mosendz, J. Pearson, F. Fradin, S. Bader, A. Hoffmann, Appl. Phys. Lett. 96 (2010) 022502.

[47] Y. Zhang, K. He, C.-Z. Chang, C.-L. Song, L.-L. Wang, X. Chen, J.-F. Jia, Z. Fang, X. Dai, W.-Y. Shan, et al., Nature Phys. 6 (2010) 584-588.

[48] G. Wu, H. Chen, Y. Sun, X. Li, P. Cui, C. Franchini, J. Wang, X.-Q. Chen, Z. Zhang, Sci. Rep. 3 (2013) 1233. 